\title{
Research Paper: The Protective Effect of Kolaviron on Molecular, Cellular, and Behavioral Characterization of Cerebellum in the Rat Model of Demyelinating Diseases
}

\author{
Gabriel Olaiya Omotoso $^{1}$ (D), Leviticus Oghenevurinrin Arietarhire ${ }^{1} \mathbb{D}$, Ileje Inelo Ukwubile ${ }^{1}$ (D), Ismail Temitayo Gbadamosi ${ }^{1}$ (D)
}

1. Department of Anatomy, Faculty of Basic Medical Sciences, College of Health Sciences, University of Ilorin, Ilorin, Nigeria.

\begin{tabular}{|c|c|}
\hline $\begin{array}{l}\text { Use your device to scan } \\
\text { and read the article online }\end{array}$ & Ctet on: Olaiya Omotoso, G., Oghenevurinrin Arietarhire, L., Inelo Ukwubile, I., \& Temitayo Gbadamosi, I. (2020). The \\
\hline 口ifh & Protective Effect of Kolaviron on Molecular, Cellular, and Behavioral Characterization of Cerebellum in the Rat Model of \\
\hline 7 & Demyelinating Diseases. Basic and Clinical Neuroscience, 11(5), 609-618. http://dx.doi.org/10.32598/bcn.9.10.300 \\
\hline 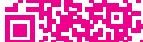 & dolihttp://dx.doi.org/10.32598/bcn.9.10.300 \\
\hline
\end{tabular}

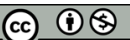

Article info:

Received: 21 Mar 2019

First Revision: 10 Apr 2019

Accepted: 27 Nov 2019

Available Online: 01 Sep 2020

Keywords:

Demyelinating disease, Cerebellum, Cuprizone, Kolaviron, Oxidative stress

\begin{abstract}
A B S T RA C T
Introduction: This study aimed at assessing the protective mechanisms of Kolaviron (KV) on the cerebellum in a rat model of demyelination.

Methods: Twenty-eight male Wistar rats were used in the present study. They were randomly divided into 4 groups of 7 rats. Group A (control) received corn oil $(0.5 \mathrm{~mL} / \mathrm{kg} / \mathrm{d}$ ); group B received $0.2 \%$ Cuprizone (CPZ); group C was treated with $200 \mathrm{mg} / \mathrm{kg} / \mathrm{d}$ of $\mathrm{KV}$, and group D received $0.2 \% \mathrm{CPZ}$ and $200 \mathrm{mg} / \mathrm{kg} / \mathrm{d} \mathrm{KV}$ for 6 weeks. CPZ powder was mixed with the regular diet while $\mathrm{KV}$ was dissolved in corn oil and administered orally. A behavioral test was conducted at the termination of the experiment. Thereafter, the animals were sacrificed and their brains were removed with the excision of the cerebellum. A part of the cerebelli underwent tissue processing with a series of $5 \mu \mathrm{m}$ thick sections cut from paraffin blocks for histological and immunohistochemical assessment. Besides, the remaining cerebellar tissues were homogenized for the spectrophotometric assays of Oxidative Stress (OS) parameters.
\end{abstract}

Results: The current research findings revealed minimal weight gain following CPZ treatment, but significant weight increase in $\mathrm{KV}$-treated rats. $\mathrm{CPZ}$ treatment was associated with a reduction in the number of the line crossed, rearing frequency, rearing duration, center square entry, and center square duration; however, it increased the freezing time, i.e. significantly reversed in the KV-treated animals. Oxidative markers, such as Superoxide Dismutase (SOD) and GPx were reduced in CPZ-treated rats with elevated MDA levels. However, these data were significantly reversed by the co-administration of $\mathrm{CPZ}$ and $\mathrm{KV}$. At the tissue level, the cerebellar cortex was characterized by poorly defined layers, cryptic granules, as well as chromatolysis and pyknotic Purkinje cells with the evidence of hypertrophic astrogliosis.

Conclusion: CPZ treatment significantly depressed locomotor and exploratory activities. Furthermore, it increased OS and cerebellar toxicity. However, KV intervention significantly enhanced behavioral functions and ameliorated CPZ-induced cerebellar degeneration. Moreover, it considerably regulated OS markers in the cerebellum of the rat model of demyelinating diseases.

\footnotetext{
* Corresponding Author:

Gabriel Olaiya Omotoso, PhD.

Address: Department of Anatomy, Faculty of Basic Medical Sciences College of Health Sciences, University of Ilorin, Ilorin, Nigeria.

Tel: +234 (703) 0505707

E-mail: omotoso.go@unilorin.edu.ng; gabrielolaiya@yahoo.com
} 


\section{Highlights}

- Cuprizone (CPZ) treatment depresses locomotor and exploratory activities.

- Kolaviron prevents CPZ-induced behavioral deficit and enhances behavioral functions.

- Kolaviron mitigates cuprizone-induced cerebellar oxidative stress.

\section{Plain Language Summary}

Demyelination is a type of brain disease that involves the shedding away of myelin sheath which eventually leads to loss of axons and neurons in the brain and spinal cord. The cause of this pathological condition ranges from genetic, environment, disease infections to direct injury to the brain. The prevalence of this condition has been on the rise and it is very common among young adults. Currently, there is no cure and the need for finding putative agents for the management, treatment as well as cure is of great urgency. This research was targeted at investigating and establishing a novel compound for management and treatment of this disorder. In this study cuprizone was used to induce demyelination in Wistar rats while Kolaviron, a bioflavonoid complex found in Garcinia kola with anti-oxidative and antiinflammatory properties, was used as a treatment for demyelination. From our results we observed that Kolaviron was able to inhibit the underlying processes involved in demyelination and improved the condition of experimental animals following treatment. This study is important as it portrays and establishes Kolaviron as a potential and good candidate for the management of demyelination as it is cheap and can be made readily available for treating people with various demyelinating conditions worldwide.

\section{Introduction}

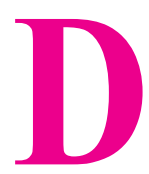

emyelination is a condition that causes the loss of myelin with the relative preservation of axons. It results from diseases that damage myelin sheaths or the myelinforming cells of the Central Nervous System (CNS), oligodendrocytes. The loss of myelin is considered as the cause of numerous CNS conditions (Love, 2006). Demyelination of focal white matter injury can occur in any location of the CNS (Ahmad, Satriotomo, Fazal, Nadeau \& Doré, 2015). The most frequent form of demyelination is observed in Multiple Sclerosis (MS). The volume of white matter lesions in the cerebellum is small, compared to other parts of the CNS; however, the lesions are detrimental to health (Ahmad et al., 2015). Recent insight into the function of the cerebellum indicates that the cerebellum not only controls movement but also impacts motor learning, cognitive behavior, as well as injury-induced sensation and adaptation due to changes in the strength of the connections among its neurons (Houk \& Miller, 2001; Diedrichsen \& Bastian, 2014). The defining clinical manifestation of MS is reported to be associated with cerebellar demyelination (Weinshenker, Issa \& Baskerville, 1996; Rot, Ledinek \& Jazbec, 2008). Despite these interesting findings, there is a paucity of information regarding the molecular, cellular, and behavioral characterization of the cerebellum in de- myelinating diseases. Understanding such characterization could help identify the underlying mechanism involved in demyelination and potential therapeutic targets.

There is a rise in the prevalence of some demyelinating diseases. Accordingly, various animal models have been used to study and find preventive measures or potential cure for these conditions. The cuprizone model, toxin-induced demyelination, has been frequently implemented; it represents a reversible demyelination and remyelination system (Kipp, Clarner, Dang, Copray \& Beyer, 2009; Faizi et al., 2016).

Cuprizone [oxalic acid bis(cyclohexylidenehydrazide)] (CPZ), is a copper chelator that induces demyelination in regions where white matter are located in the rodents' $\mathrm{CNS}$ (Sachs et al., 2014). Its underlying mechanism remains controversial; however, studies have reported that rodents fed with CPZ diet presented megamitochondria, elevated free radicals level due to Oxidative Stress (OS), uncoupling of the oxidative phosphorylation process, oligodendrocyte apoptosis, the disturbance of neurotransmitter homeostasis, synaptic dysfunction, and axonal degeneration (Kipp et al., 2009; Norkute et al., 2009; Hesse et al., 2010; Skripuletz, Gudi, Hackstette \& Stangel., 2011; Tandler \& Hoppel, 1973; Wakabayashi, Asano, \& Kurono, 1975). Although CPZ toxicity targets oligodendrocytes, other macroglia, especially astrocytes could be affected in this respect. 
Astrocytes are specialized glial cells that provide structural and functional support for neurons (Şovrea \& Boşca, 2013). They are involved in synaptogenesis, as well as regulating the communication between already formed synaptic connections (Ota, Zanetti, \& Hallock., 2013). Astrocytes participate in controlling brain homeostasis, and the intrinsic brain defense system (Kettenmann and Verkhratsky); subsequently, they make them respond in case of an insult to the brain.

Contrarily, the treatment and management of complex demyelinating diseases could be achieved through applying the phytochemical constituents of certain plants with medicinal values (Omotoso, Gbadamosi, Afolabi, Abdulwahab \& Akinlolu, 2018). Kolaviron (KV) is among such phytochemicals due to the properties attributed to it (Olaleye and Farombi, 2006; Farombi, Abarikwu, Adedara \& Oyeyemi, 2007). KV is a bioflavonoid complex isolated from the seed of Garcinia kola (bitter kola). The plant is used as an herbal remedy for treating several conditions. It provides antiviral, antibacterial, antifungal, and analgesic properties, and other activities (Olaleye \& Farombi, 2006; Farombi et al., 2007). KV inhibits the excessive production of Nitric Oxide (NO), the expression of cell death regulatory protein, and neuronal cytoskeletal dysregulation (Olajide et al., 2016). The present study aimed to assess the cellular, molecular, and behavioral changes associated with cuprizone toxicity in the cerebellum of Wistar rats, following KV intervention.

\section{Materials and Methods}

Twenty-eight male 9-week-old Wistar rats were obtained from a private animal holding (Tanke, Ilorin). The rats were housed in the Animal House of the Faculty of Basic Medical Sciences, University of Ilorin. They were allowed to acclimatize for 7 days before the onset of the experiment. They were fed on rat chow and water ad libitum. The relevant Ethical approval was obtained from the Ethics Committee of the College of Health Sciences, University of Ilorin, Nigeria. Animal handling and protocols were conducted according to the guidelines of the Ethics Committee. Garcinia kola seeds were procured from a market in Ilorin, Nigeria. Subsequently, they were verified at the herbarium of Botany Department, Faculty of Life Sciences, University of Ilorin (verification code: UILH/001/1217). CPZ was procured from Sigma-Aldrich (Germany), while PhosphateBuffered Solution (PBS; pH 7.0) was freshly prepared. Superoxide Dismutase (SOD), Glutathione Peroxidase (GPx), and Malondialdehyde (MDA) assay kits were also obtained (Abcam ${ }^{\circledR}$, USA). Rats anti-Glial Fibrillary Acidic Protein (GFAP) were acquired from Cell Signaling Technologies, Massachusetts, USA.
The methods employed in the isolation and identification of KV were as earlier described (Farombi, Shrotriya \& Surh, 2009; Olajide et al., 2017). These involved drying Garcinia kola seeds at room temperature, pulverization, extraction, as well as the assessment and confirmation of the purity and identity of the extract (KV) obtained from these processes.

The rats were randomly grouped into 4 classes, labeled as A-D (n=7/group). Group A received $0.5 \mathrm{~mL}$ of Corn Oil (CO) and served as the Control; group $\mathrm{B}$ received $0.2 \% \mathrm{CPZ}$ diet (Praet et al., 2015); group C received $200 \mathrm{mg} / \mathrm{kg}$ bw of KV (Farombi et al., 2009; Omotoso, Olajide, Gbadamosi, Rasheed \& Izuogu, 2018), and, group D received $\mathrm{KV}(200 \mathrm{mg} / \mathrm{kg} \mathrm{bw})$ and CPZ (0.2\%) diet. CO served as the vehicle in which $\mathrm{KV}$ was dissolved for the ease of oral administration. $\mathrm{CPZ}$ diet was constituted to $0.2 \%$ by mixing $0.2 \mathrm{~g} \mathrm{CPZ}$ with a $100 \mathrm{~g}$ standard rat diet. The presented treatment lasted for 42 days.

The open-field test was performed to determine the locomotor activity and exploratory behaviors of rats. Each rat was placed at the center of an open-field box (Yan et al., 2015) and tested for $10 \mathrm{~min}$; in this location, the rats usually have the freedom of movement. The activities of each rat were recorded by a video-camera situated above the area. Following the completion of the exercise, the following data were obtained: the Number of Lines Crossed (NLC), Center Squared Duration (CSD), Center Square Entry (CSE), Freezing Duration (FD), Rearing Frequency $(\mathrm{RF})$, and Rearing Duration (RD).

Sequel to last administration and behavioral study, the rats classified for histological and immunohistochemical evaluation were anesthetized using $20 \mathrm{mg} / \mathrm{kg}$ bw ketamine intraperitoneally; subsequently, they received perfused transcardially with $0.4 \mathrm{M}$ Phosphate-Buffered Saline (PBS), followed by $4 \%$ Paraformaldehyde (PFA). The brain tissues were thereafter excised, each rinsed in $0.25 \mathrm{M}$ sucrose solution thrice for $5 \mathrm{~min}$; then, post-fixed in 4\% PFA for 24 hours. The cerebelli were routinely excised and processed to obtain paraffin wax-embedded blocks. The tissues were stained using Haematoxylin and Eosin and Cresyl fast violet, as described by Fischer, Jacobson, Rose \& Zeller (2008) and Bancroft and Stevens (1982), respectively. The rats used for enzyme studies were sacrificed by cervical dislocation. Their brains were immediately removed and rinsed in $0.25 \mathrm{M}$ sucrose solution thrice for $5 \mathrm{~min}$ each; accordingly, they were placed in $30 \%$ sucrose at $4^{\circ} \mathrm{C}$

The serial sections of the cerebellum were obtained from paraffin blocks and processed as described by Olajide et al. (2017). Immunohistochemistry was performed according 


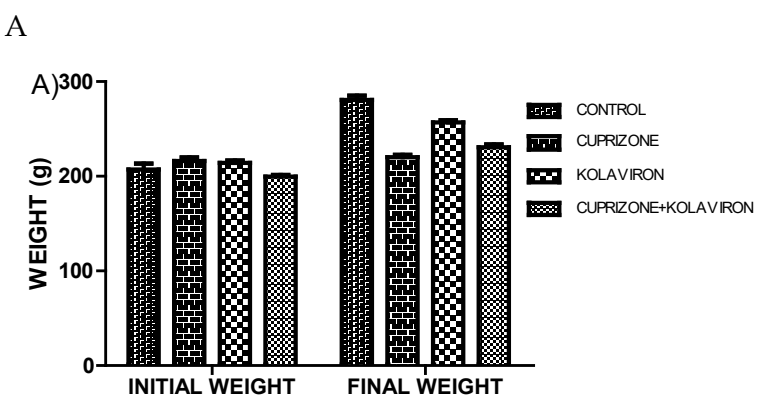

Figure 1. The weights of animals
B

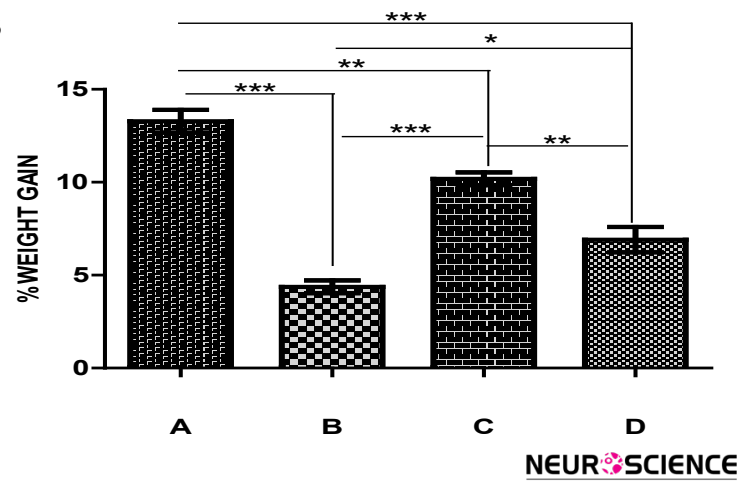

Bodyweight changes across the treated groups: A: Control; B: CPZ; C: KV; and D: CPZ plus KV;

A: Shows the initial and final weights, with all the groups having increased weight differences, though the CPZ group presented very minimal weight differences;

B: The control group (A) had the highest weight gain, while the CPZ-treated group (B) encountered the least weight gain with statistically significant differences, compared to the controls $(\mathrm{P}<0.001)$. Weight gain in the KV-treated group $(\mathrm{C})$ was also high with a statistically significant difference, compared with the controls $(\mathrm{P}<0.01)$; the differences between the KV-treated group and CPZ-treated group was statistically significant $(\mathrm{P}<0.001)$; weight gain in the $\mathrm{CPZ}+\mathrm{KV}$ group $(\mathrm{D})$ was significantly higher than the CPZ-treated group (B) $(\mathrm{P}<0.05)$, but lower than the control group $(\mathrm{A})(\mathrm{P}<0.001)$ and $\mathrm{KV}$-treated group $(\mathrm{C})(\mathrm{P}<0.01)$ Key: ${ }^{*} \mathrm{P}<0.05,{ }^{* *} \mathrm{P}<0.01$, and $\left.{ }^{* *} \mathrm{P}<0.001\right)$.

(A)

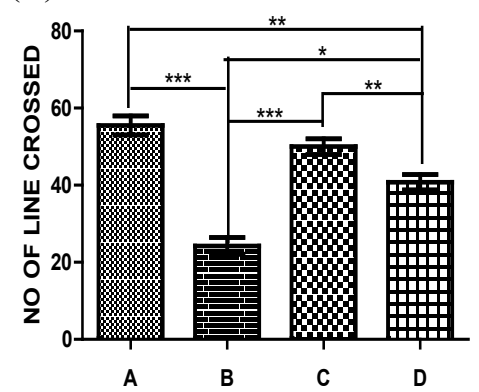

(D)

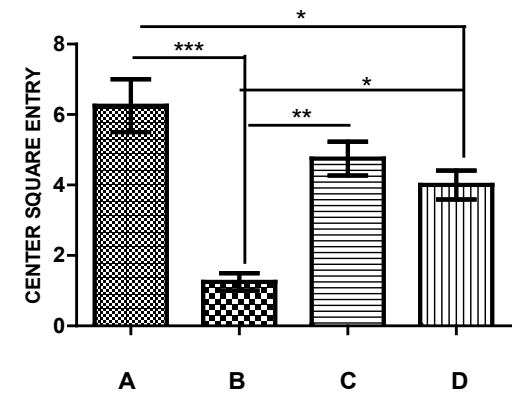

(B)

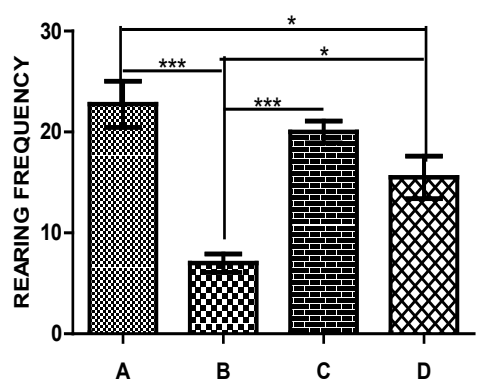

(E)

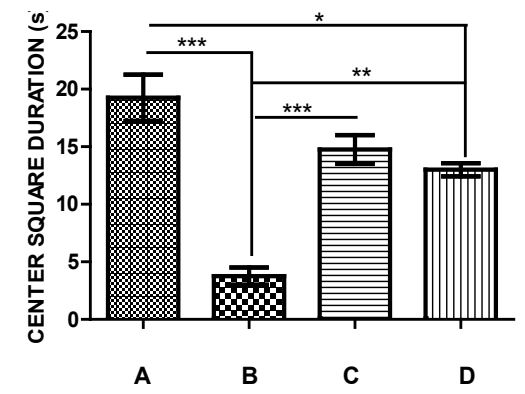

(C)

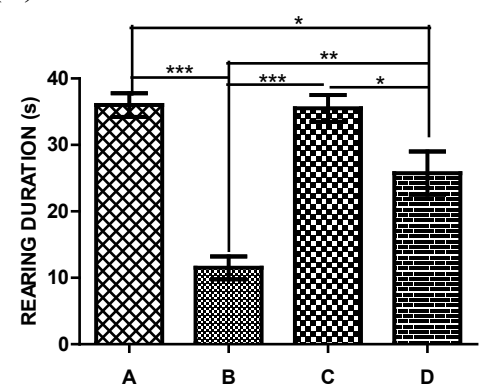

(F)

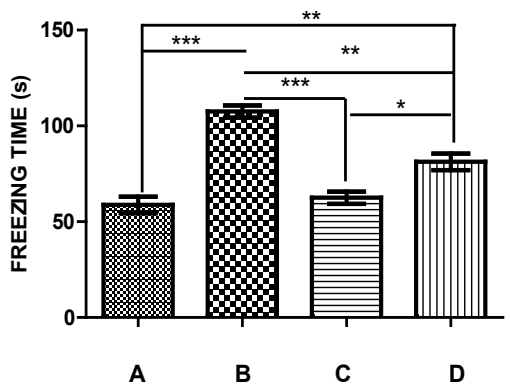

Figure 2. Behavioral tests for locomotion and exploratory activities

The locomotor and exploratory activities of rats across the research groups. A: Control; B: CPZ; C: KV; and D: CPZ and KV. In comparison with $\mathrm{CPZ}$ and $\mathrm{CPZ}+\mathrm{KV}$ treatment groups, animals given $\mathrm{KV}$ treatment experienced an increase in the number of the line crossed (A) $(\mathrm{P}<0.001 \& \mathrm{P}<0.01$, respectively), rearing frequency (B) $(\mathrm{P}<0.001$ : $\mathrm{KV}$ vs. $\mathrm{CPZ}$ groups), rearing duration (C) $(\mathrm{P}<0.001 \& \mathrm{P}<0.05$, respectively), center square entry (D) $(\mathrm{P}<0.01$ : KV vs. $\mathrm{CPZ}$ groups) and center square duration $(\mathrm{E})(\mathrm{P}<0.001$ : $\mathrm{KV}$ vs. CPZ groups). However, there was a decrease in freezing time $(\mathrm{F})$, compared with the groups solely treated with $\mathrm{CPZ}$ and KV $(\mathrm{P}<0.001 \& \mathrm{P}<0.05$, respectively). Animals simultaneously treated with $\mathrm{CPZ}$ and $\mathrm{KV}$ generally demonstrated significantly improved number of the line crossed $(A)(P<0.05)$, rearing frequency $(B)(P<0.05)$, rearing duration $(C)(P<0.01)$, center square entry $(\mathrm{D})(\mathrm{P}<0.05)$, and center square duration $(\mathrm{E})(\mathrm{P}<0.01)$, compared with the $C P Z$ group. 
(A)

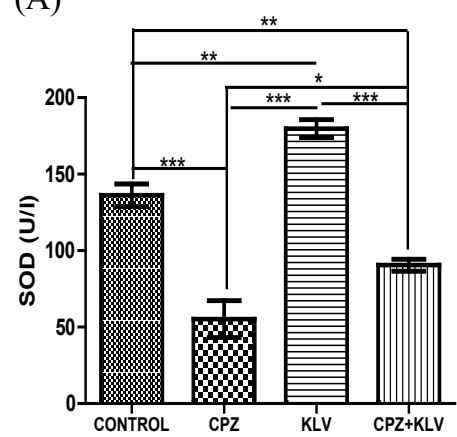

(B)

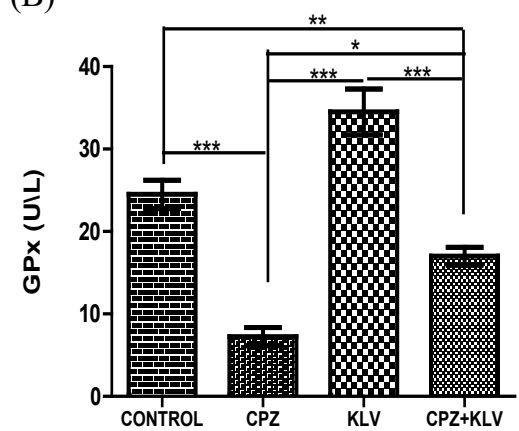

(C)

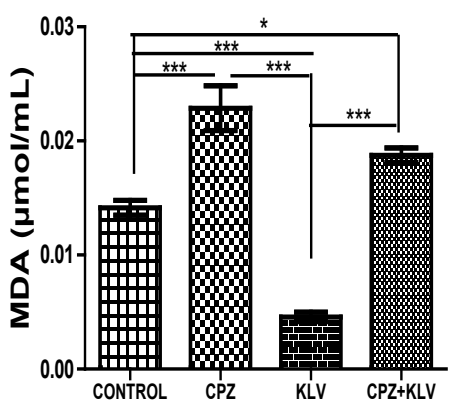

NEUROSCIENCE

Figure 3. The assessment of biochemical oxidative markers (superoxide dismutase, SOD; glutathione peroxidase, GPx \& malondialdehyde, MDA)

A: Shows statistically significant decreases in SOD activity in the CPZ-treated group (B), compared with the controls $(\mathrm{P}<0.001)$, KV-treated group $(\mathrm{C})(\mathrm{P}<0.001)$, and the $\mathrm{CPZ}+\mathrm{KV}$-treated group $(\mathrm{D})(\mathrm{P}<0.05) ; \mathrm{KV}$-treated group had the highest SOD level with statistically significant differences, compared with the control $(\mathrm{P}<0.01)$ and $\mathrm{CPZ}$-treated group $\mathrm{B}(\mathrm{P}<0.001)$; while the $\mathrm{CPZ}+\mathrm{KV}$-treated group $\mathrm{D}$ indicated a significant increase, compared to $\mathrm{CPZ}$-treated group $\mathrm{B}(\mathrm{P}<0.05)$, and a significant decrease, compared to the control $(\mathrm{P}<0.01)$ and $\mathrm{KV}$-treated groups $(\mathrm{P}<0.001)$.

B: Illustrates the activity of GPx across the study groups. GPx activity was least in the CPZ-treated group, compared to the control $(\mathrm{P}<0.001)$, KV-treated $(\mathrm{P}<0.001)$, and $\mathrm{CPZ}+\mathrm{KV}$-treated group $\mathrm{D}(\mathrm{P}<0.05)$. The co-treatment of $\mathrm{CPZ}+\mathrm{KV}$ has led to a significant increase in GPx level, compared with the $\mathrm{CPZ}$ group $(\mathrm{P}<0.05)$; though not reaching the enzyme level in the $\mathrm{KV}$ group $(\mathrm{P}<0.001)$.

C: The level of MDA was significantly elevated in the CPZ group, compared with the control $(\mathrm{P}<0.001)$ and $\mathrm{KV}$ groups $(\mathrm{P}<0.001)$; however, the simultaneous administration of $\mathrm{CPZ}$ and $\mathrm{KV}$ has led to increased MDA levels, compared to the control $(\mathrm{P}<0.05)$ and $\mathrm{KV}$ groups $(\mathrm{P}<0.001)$, which presented the least MDA level. However, the $\mathrm{CPZ}+\mathrm{KV}$ group had a lower level of MDA, compared with the CPZ-treated group, though not statistically significant.

to the method described by Goldstein and Watkins (2008). Moreover, the tissues were stained with anti-Glial Fibrillary Acidic Protein (GFAP) antibody (as a primary antibody). Next, they were treated with biotinylated secondary antibody (goat anti-rabbit) to detect the GFAP-positive cells (astrocytes) in the cerebellum. The mounted slides were viewed with the aid of an Olympus binocular research microscope (Olympus, New Jersey, USA) connected to an Amscope Camera (5.0 MP).

Cerebellar tissue homogenate was prepared with a cold $0.25 \mathrm{M}$ sucrose solution using an automated homogenizer at $4^{\circ} \mathrm{C}$. The tissue homogenate was centrifuged for $10 \mathrm{~min}$ in a microcentrifuge with a centrifugal force of $16099 \times \mathrm{g}$. The supernatants obtained were thereafter aspirated into plain bottles and analyzed for the activities of Superoxide Dismutase (SOD), Glutathione Peroxidase (GPx), and Malondialdehyde (MDA) according to the manufacturer's instruction in the biochemical kits.

All quantitative data were analyzed using GraphPad Prism. The obtained data were presented as the mean and standard error of the mean, using Analysis of Variance (ANOVA) by Tukey's multiple comparisons test. Statistical significance was also considered as $\mathrm{P}<0.05$.

\section{Results}

The feeding pattern among rats treated with $\mathrm{CPZ}$ reduced in the first two weeks of treatment; however, their feeding pattern gradually returned to normal. Additionally, that given $\mathrm{KV}$ experienced an increase in eating habits. However, after the fourth week of KV administration, their feeding habit dropped slightly. The animals treated with both $\mathrm{CPZ}$ and $\mathrm{KV}$ indicated increased feeding patterns during the early stage of administration; however, there was an observable gradual decline in their feeding pattern. Animals treated with CPZ demonstrated no appreciable weight gain (Figure 1A \& B). The group treated with KV and those that concomitantly received $\mathrm{KV}$ and $\mathrm{CPZ}$ revealed a significant weight gain, compared with the group that was treated with CPZ. However, compared with the control group, there was a reduction in their weight (Figure $1 \mathrm{~A} \& \mathrm{~B}$ ).

The open-field test data suggested that demyelinating and degenerative changes observed in CPZ decreased locomotor and exploratory activities $(\mathrm{P}<0.001)$. KV treatment was found to be protective against cuprizoneinduced behavioral deficits; it recorded an alternation in locomotor and exploratory activities, compared to the CPZ group (Figure 2). 


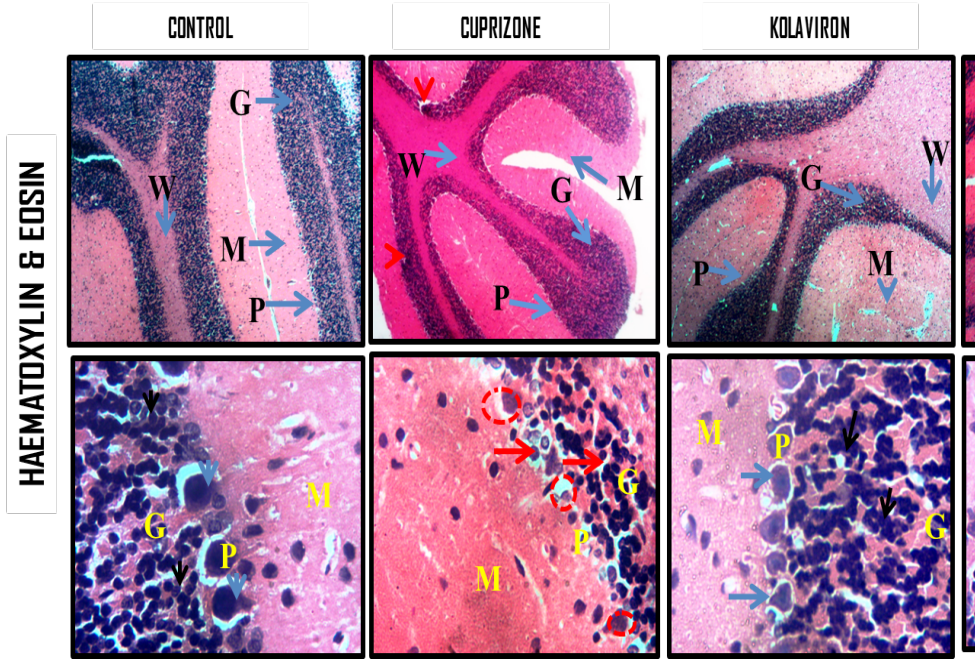

CUPRIZNE + KDLAVIRON

Figure 4. The histological demonstration of the cerebellar cortex of rats

NEUR SCIENCE

Haematoxylin and Eosin (Magnification $\times 40$ and $\times 400$ )

* Olympus binocular research microscope (Olympus, New Jersey, USA).

The representative photomicrographs of the histology of the cerebellar cortices of the studied rats. The different cortical layers were demonstrated as follows: the molecular cell layer (M), Purkinje cell layer (P), granule cell layer (G), and the medullary layer of white matter $(\mathrm{W})$. The structure of the cerebellar cortices of the control and KV-treated rats appeared relatively normal. $\mathrm{CPZ}$ treatment resulted in fragmented granule cell layers and neuropils, and cryptic granule cells, i.e. loosely arranged, degeneration of Purkinje cells and the presence of short dendrites. Neuronal morphology and cerebellar layers in rats co-treated with $\mathrm{KV}$ and CPZ appeared normal with the presence of neurons with healthy somas, axons, and dendrites. (H \& E x40 and x400).

The activities of endogenous oxidative enzymes were assessed to illustrate their involvement in CPZ-induced demyelination and understand the mechanistic inhibitory roles of KV. The spectrophotometric assay data of the SOD profile from cerebellar homogenates presented a normal SOD level in the cerebellum of the control and $\mathrm{KV}$ groups; these data were associated with a significant increase in group $\mathrm{C}$. The SOD level significantly reduced in rats intoxicated with $\mathrm{CPZ}$, compared with other groups. However, rats that concomitantly received CPZ and $\mathrm{KV}$ revealed a significant increase in SOD level, compared to the group that received CPZ (Figure 3A). The GPx level of cerebellar lysates was examined (Figure 3B). The related findings revealed that the control and KV-treated rats presented elevated GPx levels, compared to those treated with $\mathrm{CPZ}$ and adjunct $\mathrm{CPZ}$ and $\mathrm{KV}$. Furthermore, animals co-treated with $\mathrm{CPZ}$ and $\mathrm{KV}$ recorded a higher GPx level, compared with the CPZtreated rats. MDA level was also assessed to determine the degree of lipid peroxidation in cerebellar lysates in the research groups. The relevant results (Figure 3C) suggested that $\mathrm{KV}$-treated rats experienced decreased expression of MDA in the cerebellar homogenate. The CPZ-treated had increased MDA level, compared to the other groups. The rats treated concomitantly with $\mathrm{KV}$ and $\mathrm{CPZ}$ demonstrated a significant downregulation of MDA expression, compared to the CPZ-treated group.

The histochemistry of the cerebellum was demonstrated using Haematoxylin and Eosin (H \& E) and Cresyl Fast Violet (CFV) staining methods. Figure 4 shows the panoramic view and high power magnification of the microarchitecture of cerebellar cortices with distinct cell types and cell layers. The Control and KV-treated rats presented well-arranged cerebellar layers with obvious soma and dendrites; they project deep into the molecular layers, having a fan-like shape nucleus with well-stained white matter regions. Furthermore, the granular cell layers in the groups were comprised of well-arranged small granule cells. Neuronal morphology of rats intoxicated with CPZ demonstrated fragmented cerebellar layers with cryptic granules, degenerating Purkinje cells with pyknotic cell bodies, and short dendritic processes. However, the rats treated with both $\mathrm{CPZ}$ and $\mathrm{KV}$ indicated cerebellar layers and neuronal morphology, i.e. similar to that of the controls.

GFAP immunohistochemistry method was used to demonstrate astrocytic morphology and distribution in the cerebellar cortex of the explored Wistar rats. GFAP immunopositive cells within the cerebellar cortex of 


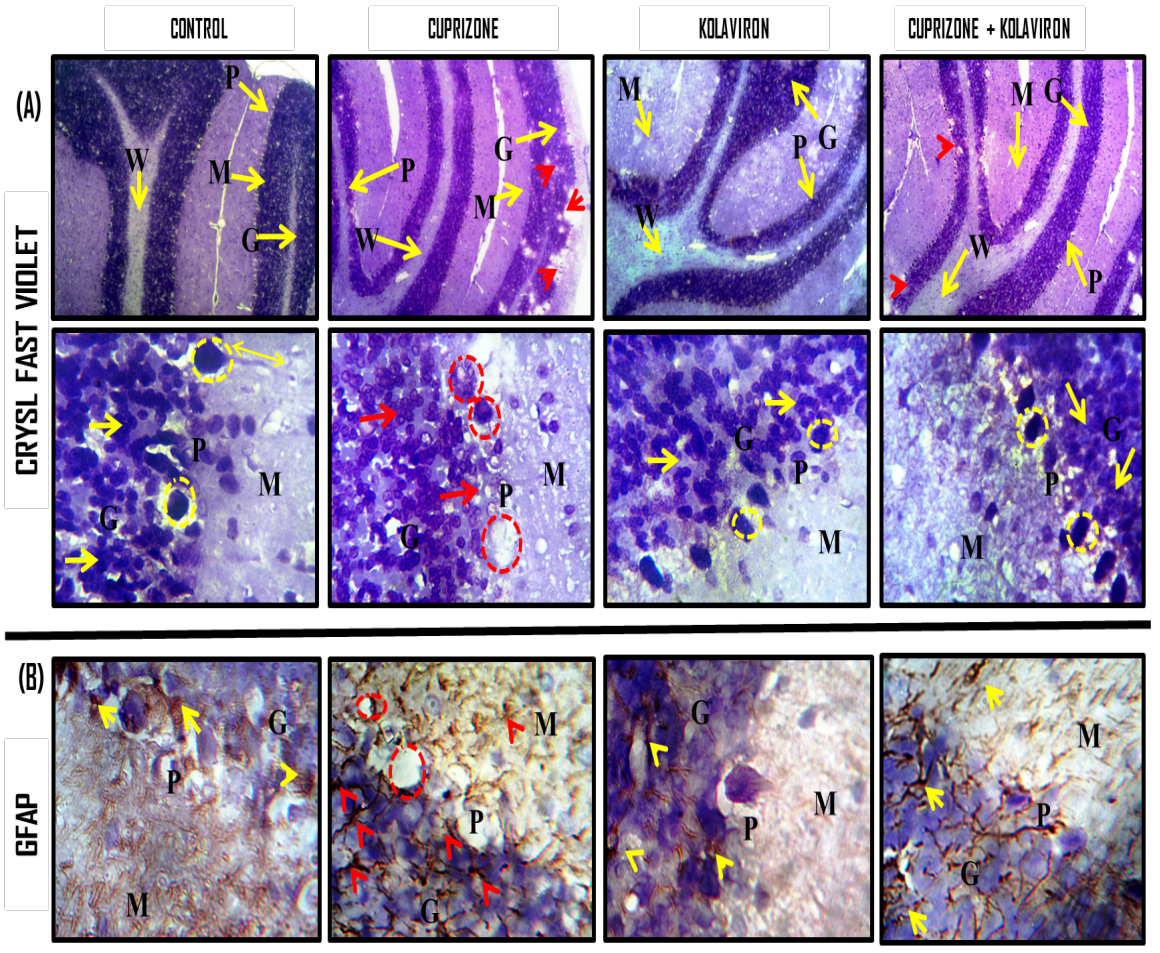

NEUR:SCIENCE

Figure 5. The histochemical demonstration of the cerebellar cortex and Immunohistochemical expression of astrocytes

A- Cresyl fast violet ((Magnification $\times 40$ and $\times 400)$; B: GFAP= glial fibrillary acidic protein immunohistochemical staining with anti-GFAP primary antibody (Magnification $\times 400)$

* Olympus binocular research microscope (Olympus, New Jersey, USA).

A \& B: Representative photomicrographs presenting the Nissl profile (A) and immunohistochemical demonstration of astrocytes (B) using CFV and anti-GFAP, respectively. Fig. 5A shows the panoramic view and high-power magnification of the cerebellar cortex stained with CFV across the research groups. The rats treated with either $\mathrm{CO}(\mathrm{A})$ or $\mathrm{KV}(\mathrm{C})$ revealed well-stained intensity and cellular density within the granular layer and white matter layer with deeply stained and well expressed Purkinje cells (yellow dotted circles), and granule cells (yellow arrows). Rats fed with CPZ (B) presented poorly stained intensity and cellular density within the granular layer with chromatolysis Purkinje cells (red dotted circles) and granule cells (red arrows). However, the studied rats treated with combined CPZ and KV experienced improved staining intensity and cellular density; also deeply stained and well expressed Purkinje cells (yellow dotted circles), and granule cells (yellow arrows). GFAP immunopositive cells;

B: (black arrows) appeared scanty around neurons in the control and KV-treated groups with relatively normal architecture. The CPZ-treated rats demonstrated evidence of increased astrocytic densities with reactive astroglia within the granule cell layer and hypertrophic cells. Meanwhile, the rats co-treated with $\mathrm{CPZ}$ and KV indicated close similarities with those of the control and KV-treated groups. (magnification $\mathrm{CFV}=\mathrm{x} 40$ and $\mathrm{x} 400$; GFAP $=x 400$ ).

the control and $\mathrm{KV}$-treated rats appeared scanty around neurons; they also were observable in between layers, with regular processes, distribution, and sizes within the neuropil. However, increased astrocytic densities with reactive astroglia within the granule cell layer and hypertrophic cells appeared within the cerebellar layers in CPZ-treated rats. Expressing astrocytes within the cerebellar cortex of rats treated with Kv and CPZ had close similarities with the control and KV-treated groups. Astrocytic processes, cellular distribution, and size were normal in the control and KV-treated rats.

\section{Discussion}

Demyelination of focal white matter injury can occur in any location of the CNS (Ahmad, Satriotomo, Fazal, Nadeau \& Doré, 2015). The most frequent form of demyelination is observed in Multiple Sclerosis (MS) Eating and diets are essential factors in controlling body weight (Drapeau et al., 2004). Studies reported reduced feeding habits during the first 2 weeks of $\mathrm{CPZ}$ consumption and gradual improvement in feeding habits post CPZ diet withdrawal (Sachs et al., 2014; Praet et al., 2015; Steel- 
man, Thompson \& Li, 2012). Therefore, it is suggested that the observed bodyweight reduction in this study was caused by reduced feeding pattern and the disruption of healthy energy flow. KV treatment significantly prevented CPZ-associated weight loss; i.e. probably caused by its ability to prevent normal energy disruption owing to its potential to mop up excess free radical. This might be the reason for the detected disruption.

As a correlative test for cellular and neuropathological changes in the cerebellar cortex in this study, the exploratory activities of rats were assessed using the open field test (Gould, Dao, \& Kovacsics, 2010).

Our results revealed a significant reduction in exploratory activities in CPZ-treated rats, corroborating a previous study by Faizi et al. (2016). However, KV significantly countered these effects. Thus, locomotor and exploratory activities depend on the intact integrity of the myelin sheath. Prior research suggested increased exploratory activities of rats following $\mathrm{KV}$ treatment after NaN3-induced neurodegeneration (Olajide et al., 2016).

Decreased SOD and GPx levels, as observed in the current study, has been implicated in the mechanism through which CPZ induces demyelination (Biancotti, Kumar \& de Vellis, 2008; Witherick, Wilkins, Scolding, \& Kemp, 2010; Praet et al., 2014). Faizi et al. (2016) reported a decrease in the SOD level following the administration of $\mathrm{CPZ}$ due to the reduced level of $\mathrm{Cu}++$, i.e. required for the proper functioning of SOD. KV treatment improved these antioxidants' levels within the cerebellar cortex of rats. SOD catalyzes the dismutation of superoxide (O2-) radical (Sun and Trumpower, 2003; Hayyan et al., 2016); if upregulated by KV, it could prevent the cytotoxic effects of $\mathrm{O} 2-$ molecules. Thus, it prevents oxidative-related damage. GPx is an enzyme capable of detoxifying Reactive Oxygen Species (ROS) and nucleophilic compounds. They could initiate lipid hydroperoxides to their corresponding alcohols and water, respectively (Muller et al., 2007). Lipid peroxidation and OS following CPZ treatment have been previously documented (Xuan et al., 2015). However, treating rats with KV significantly reduced the MDA level. Such data suggest that KV could inhibit lipid peroxidation due to its antioxidant properties.

CPZ-induced cerebellar injury revealed various degrees of structural damage to the cellular components and the normal architectural pattern of the cerebellum. These alterations in the cellular morphology of the cerebellum following CPZ treatment adversely affect signal processing. It also impacts the functionality of the syn- aptic complex, as the common features in patients with demyelinating diseases (Kutzelnigg et al., 2007; Rot et al., 2008).

Furthermore, CPZ intoxication induces megamitochondria and OS, leading to energy flow disruption and shortage (Tandler and Hoppel, 1973). The energy flow disruption and depletion alter the proper functioning of the endoplasmic reticulum, i.e. important in neuronal protein synthesis. This often causes the cellular degeneration and disintegration of oligodendrocytes perikaryon and myelin sheath (Praet et al., 2014). The neurotoxic nature of $\mathrm{CPZ}$ may explain the mechanisms by which it induces cellular degeneration and white matter demyelination. Interestingly, rats were given KV concomitantly reflected cerebellar morphology, i.e. similar to the controls. The ability of KV to restore the chromogenic nature of the Nissl substance following CPZ-induced damage might be due to its ability to prevent ER stress; this process inhibits the pathways that lead to failure of protein production owing to its antioxidative property and ROS scavenging ability.

Studies have revealed an increase in astrocytic activity following one-week CPZ intoxication (Zatta et al., 2005). The activation of astrocytes could result in extensive astrogliosis which may persist through the period of remyelination (Zaaraoui et al., 2008; Gudi et al., 2009). In the current work, changes observed on the cerebellum were characteristic of reactive astrogliosis. Accordingly, these were however absent in the cerebellar cortex of rats treated with KV. This finding suggests a cytoprotective role of $\mathrm{KV}$.

\section{Conclusion}

$\mathrm{KV}$ exhibited neuroprotection against the behavioral deficit, OS, astrogliosis, demyelination, and CPZ-induced cortical neuronal damage in adult male Wistar rats. These findings provide a lead on the potentials of $\mathrm{KV}$, as a neuroprotective agent against demyelinating diseases.

\section{Ethical Considerations}

\section{Compliance with ethical guidelines}

The Study was approved by the Ethical Review Committee, University of Ilorin (UERC/ASN/2017/969). 
Funding

This research did not receive any grant from funding agencies in the public, commercial, or non-profit sectors.

\section{Conflict of interest}

The authors declared no conflicts of interest.

\section{Acknowledgments}

The authors acknowledge the assistance and guidance of Dr. O.J. Olajide, affiliated with the Department of Anatomy, University of Ilorin.

\section{Reference}

Ahmad, A. S., Satriotomo, I., Fazal, J., Nadeau, S. E., \& Doré, S (2015). Considerations for the optimization of induced white matter injury preclinical models. Frontiers in Neurology, 6, 172. [DOI:10.3389/fneur.2015.00172] [PMID] [PMCID]

Bancroft, J.D., Stevens, A. (1982). Theory and Practice of Histological Techniques, $2^{\text {ed }}$. Edinburgh: Churchill Livingstone. https://books.google.com/books?

Biancotti, J.C., Kumar, S., de Vellis, J. (2008). Activation of inflammatory response by acombination of growth factors in cuprizone-induced demyelinated brain leadsto myelin repair. Neurochemical Research, 33(12), 2615-28. [DOI:10.1007/s11064008-9792-8] [PMID]

Diedrichsen, J., Bastian, A.J. (2014). Cerebellar function. In: M.S Gazzaniga. (Ed.), The Cognitive Neuroscience, fifth ed. Cambridge: MIT Press.

Drapeau, V., Després, J. P., Bouchard, C., Allard, L., Fournier, G., Leblanc, C., et al. (2004). Modifications in food-group consumption are related to long-term body-weight changes. Journal of Clinical Nutrition, 80(1), 29-37. [DOI:10.1093/ ajcn/80.1.29] [PMID]

Faizi, M., Salimi, A, Seydi, E., Naserzadeh, P., Kouhnavard, M. Rahimi, A., et al. (2016). Toxicity of cuprizone a Cu2p chelating agent on isolated mouse brain mitochondria: A justification for demyelination and subsequent behavioral dysfunction. Toxicology Mechanisms and Methods, 26(4), 276-83. [DOI:1 0.3109/15376516.2016.1172284] [PMID]

Farombi, E. O., Abarikwu, S. O., Adedara, I. A., Oyeyemi, M.O. (2007). Curcumin and kolaviron ameliorate di-n-butylphthalate-induced testicular damage in rats. Basic $\mathcal{E}$ Clinical Pharmacology \& Toxicology, 100(1), 43-8. [DOI:10.1111/j.17427843.2007.00005.x] [PMID]

Farombi, E.O., Shrotriya, S., Surh, Y. (2009). Kolaviron inhibits dimethyl nitrosamine-induced liver injury by suppressing cox-2 and iNOS expression via NF-ÊB and AP-1. Life Sciences 84, 149-55. [DOI:10.1016/j.lfs.2008.11.012] [PMID]
Fischer, A.H., Jacobson, K., Rose, J., Zeller, R. (2008). Hematoxylin and eosin staining of tissue and cell sections. CSH protocols, 2008, pdb.prot 4986. [DOI.:10.1101/pdb.prot4986]

Goldstein, M., Watkins, S. (2008). Immunohistochemistry. In M.A. Frederick, (Ed.), Current Protocols In Molecular Biology, (pp. 44-56), East Anglia: Wiley and Sons.

Gould, T. D., Dao, D. T., Kovacsics, C. E. (2010). The open field test. Mood and Anxiety Related Phenotypes in Mice, 42, 1-20. [DOI:10.1007/978-1-60761-303-9_1]

Gudi, V., Gingele, S., Skripuletz, T., Stangel, M (2014). Glial response during cuprizone-induced de- and remyelination in the CNS: Lessons learned. Frontiers in Cellular Neuroscience, 8 73. [DOI:10.3389/fncel.2014.00073] [PMID] [PMCID]

Gudi, V., Moharregh-Khiabani, D., Skripuletz, T., Koutsoudaki, P.N., Kotsiari, A., Skul-jec, J. (2009). Regional differences between grey and white matter in cuprizone induced demyelination. Brain Research, 1283, 127-38. [DOI:10.1016/j.brainres.2009.06.005] [PMID]

Hayyan, M., Hashim, M. A., Al-Nashef, I. M. (2016). Superoxide Ion: generation and chemical implications. Chemical Reviews, 116(5), 3029-85. [DOI:10.1021/acs.chemrev.5b00407] [PMID]

Hesse, A., Wagner, M., Held, J., Bruck, W., Salinas-Riester, G., Hao, Z. (2010). Intoxic demyelination oligodendroglial cell death occurs early and is FAS independent. Neurobiology of Disease, 37(2), 362-9. [DOI:10.1016/j.nbd.2009.10.016] [PMID] [PMCID]

Houk, J.C., Miller, L.E. (2001). Cerebellum: Movement regulation and cognitive functions. In: Encyclopedia of Life Science. Nature Publishing Group. [DOI:10.1038/npg.els.0000036]

Kettenmann, H., Verkhratsky, A. (2011). Neuroglia - living nerve glue. Fortschr Neurol Psychiatr, 79(10), 588-97. [DOI:10.1055/s-0031-1281704] [PMID]

Kipp, M., Clarner, T., Dang, J., Copray, S., Beyer, C. (2009). The cuprizone animal model: New insights into an old story. Acta Neuropathologica, 118, 723-736. [DOI:10.1007/s00401-009-05913] [PMID]

Kutzelnigg, A., Faber-Rod, J. C., Bauer, J., Lucchinetti, C. F., Sorensen, P. S., Laursen, H. (2007). Widespread demyelination in the cerebellar cortex in multiple sclerosis. Brain Pathology 17(1), 38-44. [DOI:10.1111/j.1750-3639.2006.00041.x] [PMID]

Love, S. (2006). My approach, demyelinating diseases. Journal of Clinical Pathology, 59, 1151-9. [DOI:10.1136/jcp.2005.031195] [PMID] [PMCID]

Muller, F. L., Lustgarten, M. S., Jang, Y., Richardson, A., van Remmen, H. (2007). Trends in oxidative aging theories. Free Radical Biology and Medicine, 43(4), 477-503. [DOI:10.1016/j. freeradbiomed.2007.03.034] [PMID]

Norkute, A., Hieble, A., Braun, A., Johann, S., Clarner, T., Baumgartner, W. (2009). Cuprizone treatment induces demyelination and astrocytosis in the mouse hippocampus. Journal of Neuroscience Research, 87(6), 1343-55. [DOI:10.1002/jnr.21946] [PMID]

Olajide, O. J., Enaibe, B. U., Bankole, O. O., Akinola, O. B., Laoye, B. J., Ogundele, O. M. (2016). Kolaviron was protective agains sodium azide (NaN3)-induced oxidative stress in the prefrontal cortex. Metabolic Brain Disease, 31(1), 25-35. [DOI:10.1007/ s11011-015-9674-0] [PMID] 
Olajide, O. J., Ugbosanmi, A. T., Enaibe, B. U., Ogunrinola, K. Y., Lewu, S.F., Asogwa, N. T., et al. (2017). Cerebellar molecular and cellular characterization in rat models of Alzheimer's disease: Neuroprotective mechanisms of Garcinia biflavonoid complex.

Annals of Neurosciences, 24(1), 32-45. [DOI:10.1159/000464421] [PMID] [PMCID]

Olaleye, S. B., \& Farombi, E. O. (2006). Attenuation of indomethacin- and $\mathrm{HCl} /$ ethanol-induced oxidative gastric mucosa damage in rats by kolaviron, a natural biflavonoid of Garcinia kola seed. Phytotherapy Research, 20, 14-20. [DOI:10.1002/ ptr.1793] [PMID]

Omotoso, G. O., Gbadamosi, I. T., Afolabi, T. T., Abdulwahab A. B., Akinlolu, A. A. (2018). Ameliorative effects of Moringa on cuprizone-induced memory decline in rat model of multiple sclerosis. Anatomy and Cell Biology, 51, 119-27. [DOI:10.5115/ acb.2018.51.2.119] [PMID] [PMCID]

Omotoso, G. O., Olajide, O. J., Gbadamosi, I. T., Rasheed, M. A., Izuogu, C. T. (2018). Kolaviron protects the prefrontal cortex and hippocampus against histomorphological and neurobehavioral changes in cuprizone model of multiple sclerosis. Malaysian Journal of Medical Sciences, 25(2), 50-63. [DOI:10.21315/mims2018.25.2.6] [PMID] [PMCID]

Ota, Y., Zanetti, A. T., \& Hallock, R. M. (2013). The role of astrocytes in the regulation of synaptic plasticity and memory formation. Neural Plasticity, 2013. [DOI:10.1155/2013/185463] [PMID] [PMCID]

Praet, J., Guglielmetti, C., Berneman, Z., van der Linden, A., Ponsaerts, P. (2014). Cellular and molecular neuropathology of the cuprizone mouse model: Clinical relevance for multiple sclerosis. Neuroscience \& Biobehavioral Reviews, 47, 485-505. [DOI:10.1016/j.neubiorev.2014.10.004] [PMID]

Praet, J., Orije, J., Kara, F., Guglietmetti, C., Santermans, E. Daans, J., et al. (2015). Cuprizone-induced demyelination and demyelination-associated inflammation result in different proton magnetic resonance metabolite spectra. NMR in Biomedicine, 28(4), 505-513. [DOI:10.1002/nbm.3277] [PMID] [PMCID]

Rot, U., Ledinek, A. H., \& Jazbec, S. Š. (2008). Clinical, magnetic resonance imaging, cerebrospinal fluid and electrophysiological characteristics of the earliest multiple sclerosis. Clinical Neurology and Neurosurgery, 110(3), 233-8. [DOI:10.1016/j. clineuro.2007.11.001] [PMID]

Sachs, H. H., Bercury, K. K., Popescu, D. C., Narayanan, S. P., \& Macklin, W. B. (2014). A new model of cuprizonemediated demyelination/remyelination. ASN Neuro, 6(5), 1759091414551955. [DOI:10.1177/1759091414551955] [PMID] [PMCID]

Skripuletz, T., Gudi, V., Hackstette, D., \& Stangel, M. (2011). De-and remyelination in the CNS white and grey matter induced by cuprizone: The old, the new, and the unexpected. Histology and Histopathology, 26(12), 1585-97. https://digitum.um.es/digitum/bitstream/10201/49540/1/Skripuletz-26-1585-1597-2011.pdf

Sovrea, A. S., \& Boşca, A. B. (2013). Astrocytes reassessment - an evolving concept part one: Embryology, biology, morphology and reactivity. Journal of Molecular Psychiatry, 1(1), 18 [DOI:10.1186/2049-9256-1-18] [PMID] [PMCID]
Steelman, A., Thompson, J. P., Li, J. (2012). Demyelination and remyelination in anatomically distinct regions of the corpus callosum following cuprizone intoxication. Neuroscience Research, 72(1), 32-42. [DOI:10.1016/j.neures.2011.10.002] [PMID] [PMCID]

Sun, J., \& Trumpower, B. L. (2003). Superoxide anion generation by the cytochrome bc1 complex. Archives of Biochemistry and Biophysics, 419(2), 198-206. [DOI:10.1016/j.abb.2003.08.028] [PMID]

Tandler, B., \& Hoppel, C. L. (1973). Division of giant mitochondria during recovery from cuprizone intoxication. The Journal of Cell Biology, 56(1), 266-72. [DOI:10.1083/jcb.56.1.266] [PMID] [PMCID]

Wakabayashi, T., Asano, M., \& Kurono, C. (1975). Mechanism of the formation of megamitochondria induced by copperchelating agents, II; Isolation and some properties of megamitochondria from the cuprizone-treated mouse liver. Pathology International, 25(1), 39-49. [DOI:10.1111/j.1440-1827.1975. tb00148.x]

Weinshenker, B. G., Issa, M., \& Baskerville, J. (1996). Long-term and short-term outcome of multiple sclerosis: A 3-year followup study. Archives of Neurology, 53(4), 353-358. [DOI:10.1001/ archneur.1996.00550040093018] [PMID]

Witherick, J., Wilkins, A., Scolding, N., \& Kemp, K. (2010). Mechanisms of oxidative damage in multiple sclerosis and a cell therapy approach to treatment. Autoimmune diseases, 2011. [DOI:10.4061/2011/164608] [PMID] [PMCID]

Xuan, Y., Yan, G., Wu, R., Huang, Q., Li, X., \& Xu, H. (2015). The cuprizone-induced changes in 1H-MRS metabolites and oxidative parameters in C57BL/ 6 mouse brain: Effects of quetiapine. Neurochemistry International, 90, 185-92. [DOI:10.1016/j. neuint.2015.08.015] [PMID]

Yan, G., Xuan, Y., Dai, Z., Shen, Z., Zhang, G., Xu, H., \& Wu, R. (2015). Brain metabolite changes in subcortical regions after exposure to cuprizone for 6 weeks: Potential implications for schizophrenia. Neurochemical Research, 40(1), 49-58. [DOI:10.1007/s11064-014-1464-2] [PMID]

Zaaraoui, W., Deloire, M., Merle, M., Girard, C., Raffard, G., Biran, M., ... \& Franconi, J. M. (2008). Monitoring demyelination and remyelination by magnetization transfer imaging in the mouse brain at 9.4 T. Magnetic Resonance Materials in Physics, Biology and Medicine, 21(5), 357-62. [DOI:10.1007/s10334-0080141-3] [PMID] [PMCID]

Zatta, P., Raso, M., Zambenedetti, P., Wittkowski, W., Messori, L., \& Piccioli, F., et al. (2005). Copper and zinc dismetabolism in the mouse brain upon chronic cuprizone treatment. Cellular and Molecular Life Sciences CMLS, 62(13), 1502-13. [DOI:10.1007/s00018-005-5073-8] [PMID] 